\title{
The effect of van der Waals-based interface cohesive law on carbon nanotube-reinforced composite materials
}

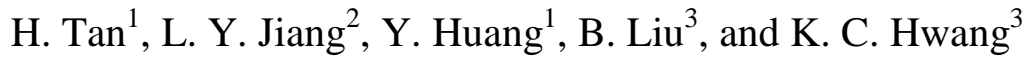 \\ ${ }^{1}$ Department of Mechanical Science and Engineering, University of Illinois at Urbana-Champaign, \\ Urbana, IL 61801 \\ ${ }^{2}$ Department of Mechanical and Materials Engineering, University of Western Ontario, London, ON N6A \\ 5B9, Canada \\ ${ }^{3}$ Department of Engineering Mechanics, Tsinghua University, Beijing 100084, China
}

\begin{abstract}
:
The nonlinear cohesive law derived from the weak van der Waals force for carbon nanotube/polymer interfaces is incorporated in present study of CNT-reinforced composites. Carbon nanotubes can indeed improve the mechanical behavior of composite at the small strain, but such improvement disappears at relatively large strain because the completely debonded nanotubes behave like voids in the matrix and may even weaken the composite. The increase of interface adhesion between carbon nanotubes and polymer matrix may significantly improve the composite behavior at the large strain.
\end{abstract}

Keywords: C. van der Waals force; B. Fibre/matrix bond; A. Carbon fibres; B. Interface; A. Polymer-matrix composites (PMCs) 


\section{Introduction}

Carbon nanotubes (CNT) display superior mechanical properties, and are an excellent candidate of reinforcements for nanocomposites [1,2]. The CNT-reinforced composites, however, never reach their expected mechanical properties [3]. This has triggered significant research efforts to understand this shortfall and to improve the mechanical properties of CNT-reinforced nanocomposites [4-9].

Nanocomposites possess a large amount of interfaces due to the small (nanometer) size of reinforcements. The interface behavior can significantly affect the mechanical properties of nanocomposites. For example, carbon nanotubes in general do not bond well to polymers [1012], and their interactions result mainly from the weak van der Waals forces [13-17]. Consequently CNTs may slide inside the matrix and may not provide much reinforcing effect. It is, however, important to assess whether the poor interface behavior is indeed responsible for the short fall of CNT-reinforced composites in order to reach their expected properties.

It is difficult to account for the van der Waals interactions in the continuum modeling of nanocomposites. Jiang et al. [2] established a nonlinear cohesive law for the CNT/polymer interfaces directly from the Lennard-Jones $(\mathrm{LJ})$ potential for the van der Waals interactions. The

cohesive law gives analytically the normal cohesive stress at the CNT/polymer interface, $\sigma^{\text {int }}$, in terms of the interface opening displacement $[u]$. The normal cohesive stress $\sigma^{\text {int }}$ displays nonlinear hardening, peak strength, and softening behavior as the opening displacement $[u]$ increases. All cohesive law properties (e.g., cohesive strength, cohesive energy) are obtained analytically in terms of the parameters in the LJ potential.

The objective of this paper is to incorporate Jiang et al.'s [2] nonlinear cohesive law for $\mathrm{CNT} /$ polymer interfaces in the study of the macroscopic behavior of CNT-reinforced composites. It provides a direct link between the macroscopic behavior of nanocomposites and the nanoscale behavior of interfaces, and therefore an assessment whether the interface behavior is indeed responsible for the macroscopic properties of nanocomposites. 
The paper is outlined in the following. The nonlinear cohesive law [2] for CNT/polymer interfaces is summarized in Section 2. A micromechanics model is provided in Section 3 to predict the nonlinear behavior of CNT-reinforced composite in terms of the properties of CNT/matrix interfaces, as well as those of CNTs and matrix. An example of a CNT-reinforced composite subjected to hydrostatic tension is used to illustrate the effect of nonlinear cohesive law. The nonlinear stress-strain curve of the CNT-reinforced composite is given in Section 4.

\section{A Nonlinear Cohesive Law for Carbon Nanotube/Polymer Interfaces}

The energy between two atoms of distance $r$ due to the van der Waals force can be represented by the LJ 6-12 potential,

$$
V(r)=4 \varepsilon\left(\frac{\sigma^{12}}{r^{12}}-\frac{\sigma^{6}}{r^{6}}\right)
$$

where $\sqrt[6]{2} \sigma$ is the equilibrium distance between atoms, and $\varepsilon$ is the bond energy at the equilibrium distance. For the van der Waals interaction between a carbon atom and a $-\mathrm{CH}_{2}-$ unit of polyethylene, $\varepsilon=0.004656 \mathrm{ev}$ and $\sigma=0.3825 \mathrm{~nm}[18]$.

Based on the LJ potential for the van der Waals interaction, Jiang et al. [2] established the following cohesive law for CNT/polymer interfaces,

$$
\sigma^{\mathrm{int}}=3.07 \sigma_{\max }\left[\left(1+0.682 \frac{\sigma_{\max }}{\Phi_{\text {total }}}[u]\right)^{-4}-\left(1+0.682 \frac{\sigma_{\max }}{\Phi_{\text {total }}}[u]\right)^{-10}\right],
$$

where $\sigma^{\text {int }}$ and $[u]$ are the normal cohesive stress and opening displacement at the CNT/polymer

interface, respectively, the cohesive strength $\sigma_{\max }$ and total cohesive energy $\Phi_{\text {total }}$ are related to the parameters $\sigma$ and $\varepsilon$ in $\mathrm{LJ}$ potential in Eq. (2.2) by

$$
\sigma_{\max }=\frac{6 \pi}{5} \rho_{p} \rho_{c} \varepsilon \sigma^{2}
$$


and

$$
\Phi_{\text {total }}=\frac{4 \pi}{9} \sqrt{\frac{5}{2}} \rho_{p} \rho_{c} \varepsilon \sigma^{3} .
$$

Here $\rho_{c}$ is the CNT area density (number of carbon atoms per unit area of CNT) which equals $3.82 \times 10^{19} \mathrm{~m}^{-2}$ [19], $\rho_{p}$ is the polymer volume density (number of polymer molecules per unit volume). For the $-\mathrm{CH}_{2}$ - unit in polyethylene, $\rho_{p}$ can be obtained from the ratio of polyethylene mass density $0.71 \times 10^{3} \mathrm{~kg} \cdot \mathrm{m}^{-3} \quad$ [18] to the mass of $-\mathrm{CH}_{2^{-}}$unit $\left(2.3 \times 10^{-26} \mathrm{~kg}\right)$ as $\rho_{p}=3.1 \times 10^{28} \mathrm{~m}^{-3}$. Equation (2.3) then gives a rather high cohesive strength, $\sigma_{\max }=479 \mathrm{MPa}$, as compared to the Young's modulus of polyethylene ( 0.9GPa). However, Eq. (2.4) gives a very low cohesive energy $\Phi_{\text {total }}=0.107 \mathrm{Jm}^{-2}$, which is consistent with the poor bonding between CNTs and polymer. Figure 1 shows the normal cohesive stress $\sigma^{\text {int }}$ versus the interface opening displacement $[u]$. The cohesive stress increases rapidly at small opening displacement, reaches the cohesive strength at $[u]=0.0542 \mathrm{~nm}$ and then gradually decreases as $[u]$ increases. The overall area underneath the curve is the total cohesive energy, $0.107 \mathrm{Jm}^{-2}$.

\section{A Micromechanics Model for Nanocomposites with Nonlinear Interface Cohesive Law}

We first consider a composite material with long fibers since CNTs have very large aspect ratio (e.g., $10^{5}$ ). The fiber volume fraction is denoted by $f$.

\subsection{Macroscopic strain of the nanocomposite}

The macroscopic stress $\bar{\sigma}$ and strain $\bar{\varepsilon}$ of the nanocomposite represent the collective, homogenized behavior of fibers and matrix. They are distinguished from the microscopic stress 
$\sigma$ and strain $\varepsilon$ in fibers and matrix since the latter satisfy the constitutive laws in the corresponding constituents, and are nonuniform due to the material inhomogeneities.

The macroscopic strain is given by $[20,21]$

$$
\bar{\varepsilon}=\boldsymbol{M}_{\mathrm{m}}: \overline{\boldsymbol{\sigma}}+f\left[\left(\boldsymbol{M}_{f}-\boldsymbol{M}_{\mathrm{m}}\right):\left\langle\boldsymbol{\sigma}^{f}\right\rangle+\left\langle\varepsilon^{\mathrm{int}}\right\rangle\right]
$$

where the matrix and fibers are linear elastic with the elastic compliance tensor $\boldsymbol{M}_{m}$ for the matrix and $\boldsymbol{M}_{f}$ for fibers; $\left\langle\boldsymbol{\sigma}^{f}\right\rangle$ is the average stress in fibers; and $\left\langle\varepsilon^{\text {int }}\right\rangle$ represents the contribution from the fiber/matrix interface and is given by

$$
\left\langle\varepsilon^{\mathrm{int}}\right\rangle=\frac{1}{2 \Omega_{f}} \int_{S_{\mathrm{int}}}([\boldsymbol{u}] \otimes \boldsymbol{n}+\boldsymbol{n} \otimes[\boldsymbol{u}]) d A
$$

Here $\Omega_{f}$ is the fiber volume, and $S_{\text {int }}$ represents all fiber/matrix interfaces. For perfectly bonded interfaces, $\left\langle\varepsilon^{\mathrm{int}}\right\rangle=\mathbf{0}$. For not-well-bonded interfaces as in CNT-reinforced composites, $\left\langle\varepsilon^{\text {int }}\right\rangle$ and $\left\langle\sigma^{f}\right\rangle$ are to be determined from the interface cohesive law.

We consider a simple case of an isotropic matrix containing randomly distributed, long and isotropic fibers subjected to hydrostatic tension $\overline{\boldsymbol{\sigma}} \boldsymbol{I}$, where $\boldsymbol{I}$ is the second-order identity tensor. The macroscopic strain of the composite is $\bar{\varepsilon} \boldsymbol{I}$, and $\bar{\varepsilon}$ is obtained from Eq. (3.1) as

$$
\bar{\varepsilon}=\frac{1}{3 K_{m}} \bar{\sigma}+\frac{f}{3}\left[\left(\frac{1}{3 K_{f}}-\frac{1}{3 K_{m}}\right)\left\langle\sigma_{k k}^{f}\right\rangle+\left\langle\varepsilon_{k k}^{\mathrm{int}}\right\rangle\right],
$$

where $K_{m}$ and $K_{p}$ are the elastic bulk moduli of the matrix and fibers, respectively, and $\left\langle\varepsilon_{k k}^{\text {int }}\right\rangle$ is related to the displacement jump $[u]$ in the normal direction across the fiber/matrix interface by

$$
\left\langle\varepsilon_{k k}^{\mathrm{int}}\right\rangle=\frac{1}{\Omega_{f}} \int_{S_{\mathrm{int}}}[u] d A
$$


For the nonlinear cohesive law in Section 2, the displacement jump $[u]$ is related to the normal cohesive stress $\sigma^{\text {int }}$ via Eq. (2.2). A micromechanics model, such as the dilute solution in the next section, is needed to determine $\left\langle\sigma_{k k}^{f}\right\rangle$ and $\left\langle\varepsilon_{k k}^{\text {int }}\right\rangle$ in terms of $\bar{\sigma}$ (or $\bar{\varepsilon}$ ). Equation (3.3) then gives the nonlinear stress-strain relation.

\subsection{Dilute Solution}

Since the CNT volume fraction is small, we may use the dilute solution which neglects the interactions among fibers. Consider a long fiber of radius $R$ in an infinite matrix subjected to remote hydrostatic tension. Let $\bar{\sigma}$ and $\bar{\varepsilon}$ denote the macroscopic stress and strain of the composite, and $\sigma^{\text {int }}$ the normal cohesive stress at the fiber/matrix interface. The stresses in the fiber are uniform, $\sigma_{r r}^{f}=\sigma_{\theta \theta}^{f}=\sigma^{\mathrm{int}}=$ constant, and $\sigma_{z z}^{f}=$ constant, where $(r, \theta, z)$ are the cylindrical coordinates. The strains in the fiber are $\varepsilon_{r r}^{f}=\varepsilon_{\theta \theta}^{f}=\left[\left(1-\nu_{f}\right) \sigma^{\text {int }}-\nu_{f} \sigma_{z z}^{f}\right] / E_{f}$ and $\varepsilon_{z z}^{f}=\left(\sigma_{z z}^{f}-2 v_{f} \sigma^{\text {int }}\right) / E_{f}$, where $E_{f}$ and $v_{f}$ are the Young's modulus and Poisson's ratio of the fiber. The radial displacement at the fiber boundary is $R\left[\left(1-v_{f}\right) \sigma^{\mathrm{int}}-\mathrm{v}_{f} \sigma_{z z}^{f}\right] / E_{f}$.

The matrix stress in the fiber direction is the same as macroscopic stress $\bar{\sigma}$. The radial displacement in the matrix takes the form $u_{r}=A r+B / r$ [22], where the coefficients $A$ and $B$ are to be determined by the remote boundary condition $\left.\sigma_{r r}^{m}\right|_{r \rightarrow \infty}=\bar{\sigma}$ and the interface condition $\left.\sigma_{r r}^{m}\right|_{r=R}=\sigma^{\text {int }}$. The displacement at the fiber/matrix interface $r=R$ is then given by $R\left[\left(2-v_{m}\right) \bar{\sigma}-\left(1+v_{m}\right) \sigma^{\text {int }}\right] / E_{m}$, where $E_{m}$ and $v_{m}$ are the Young's modulus and Poisson's ratio of the matrix, respectively.

The opening displacement is the displacement jump across the fiber/matrix interface, i.e.,

$$
[u]=\frac{R}{E_{m}}\left[\left(2-v_{m}\right) \bar{\sigma}-\left(1+v_{m}\right) \sigma^{\mathrm{int}}\right]-\frac{R}{E_{f}}\left[\left(1-v_{f}\right) \sigma^{\mathrm{int}}-v_{f} \sigma_{z z}^{f}\right]
$$


It is recalled that the normal cohesive stress $\sigma^{\mathrm{int}}=\sigma^{\mathrm{int}}([u])$ is related to the opening displacement $[u]$ via the nonlinear cohesive law (2.2). Equation (3.5) then gives the macroscopic stress $\bar{\sigma}$ in terms of $[u]$ and $\sigma_{z z}^{f}$, and $\sigma_{z z}^{f}$ is to be determined by the Reuss approximation in the following. The macroscopic strain $\bar{\varepsilon}$ can be obtained from Eq. (3.3).

For very long fibers, Reuss approximation [23] gives the fiber stress $\sigma_{z z}^{f}$ in the fiber direction to be the same as the macroscopic stress

$$
\sigma_{z z}^{f}=\bar{\sigma}
$$

The Reuss approximation, however, gives a lower bound solution and underestimates the reinforcing effect of fibers.

The average stress in fiber is $\left\langle\sigma_{k k}^{f}\right\rangle=2 \sigma^{\text {int }}+\bar{\sigma}$. The additional strain $\left\langle\varepsilon_{k k}^{\text {int }}\right\rangle$ due to interface opening is $2[u] / R$. Equation (3.5) gives the macroscopic stress in terms of the interface opening displacement $[u]$ as

$$
\bar{\sigma}=\frac{\frac{[u]}{R}+\left(\frac{1+v_{m}}{E_{m}}+\frac{1-v_{f}}{E_{f}}\right) \sigma^{\mathrm{int}}([u])}{\frac{2-v_{m}}{E_{m}}+\frac{v_{f}}{E_{f}}}
$$

where $\sigma^{\text {int }}([u])$ is obtained from Eq. (2.2). The macroscopic strain is obtained in terms of $[u]$ from Eq. (3.3) as

$$
\bar{\varepsilon}=\frac{1}{3 K_{m}} \bar{\sigma}+\frac{f}{3}\left\{\left(\frac{1}{3 K_{f}}-\frac{1}{3 K_{m}}\right)\left[2 \sigma^{\mathrm{int}}([u])+\bar{\sigma}\right]+2 \frac{[u]}{R}\right\}
$$


Equations (3.7) and (3.8) give the macroscopic stress $\bar{\sigma}$ and strain $\bar{\varepsilon}$ in terms of $[u]$ based on the Reuss approximation, and therefore provide the nonlinear stress-strain relation of the CNTreinforced composite.

\section{Stress-strain curve of CNT-reinforced composite}

The CNT Young's modulus $E_{f}$ is on the order of 1TPa, which is several orders of magnitude higher than the modulus $E_{m}$ of polymer matrix (e.g., $0.9 \mathrm{GPa}$ for polyethylene). (The CNT elastic modulus in the CNT radial direction may be smaller than that in the axial direction, but is still much larger than the polymer modulus.) For $E_{f} \gg E_{m}$, the stress-strain relation (3.7) and (3.8) is simplified to

$$
\begin{gathered}
\bar{\sigma}=\frac{E_{m}}{2-v_{m}} \frac{[u]}{R}+3.07 \frac{1+v_{m}}{2-v_{m}} \sigma_{\max }\left[\left(1+0.682 \frac{\sigma_{\max }}{\Phi_{\text {total }}}[u]\right)^{-4}-\left(1+0.682 \frac{\sigma_{\text {max }}}{\Phi_{\text {total }}}[u]\right)^{-10}\right], \\
\bar{\varepsilon}=\frac{1-2 v_{m}+f}{2-v_{m}} \frac{[u]}{R} \\
+3.07\left[1+v_{m}-\frac{f}{3}\left(5-v_{m}\right)\right] \frac{1-2 v_{m}}{2-v_{m}} \frac{\sigma_{\max }}{E_{m}}\left[\left(1+0.682 \frac{\sigma_{\max }}{\Phi_{\text {total }}}[u]\right)^{-4}-\left(1+0.682 \frac{\sigma_{\text {max }}}{\Phi_{\text {total }}}[u]\right)^{-10}\right],
\end{gathered}
$$

where Eq. (2.2) has been used.

Figure 2 shows the stress-strain curve for a CNT-reinforced polyethylene composite subjected to hydrostatic tension. For comparison the straight line for the linear elastic polyethylene matrix is also shown. The polyethylene Young's modulus and Poisson's ratio are $E_{m}=0.9 \mathrm{GPa}$ and $v_{m}=0.33$, respectively. The nanotube radius is $R=1.25 \mathrm{~nm}$ for the armchair 
$(18,18)$ CNTs. The CNT volume fraction is $f=10 \%$. For strain less than $13.9 \%$, the stress-strain curve is almost (but not exactly) a straight line that is above the straight line for the matrix and reflects the CNT reinforcing effect. (The curve for the composite is not significantly higher than the straight line for the polyethylene matrix because the Reuss approximation gives a lower bound solution and therefore underestimates the CNT reinforcing effect.) Once the strain reaches $13.9 \%$ (and the stress reaches the peak strength $406 \mathrm{MPa}$ ), both stress and strain decrease due to the interface softening behavior displayed in the cohesive law (Fig. 1). The stress and strain start to increase again as the strain drops to $9.1 \%$, but the curve becomes lower than the straight line for the matrix because the completely debonded CNTs are equivalent to voids and therefore weaken the matrix. For the displacement-controlled experiment, the stress-strain curve would drop vertically from $406 \mathrm{MPa}$ to $289 \mathrm{MPa}$ at the $13.9 \%$ strain, as illustrated by the arrow in Fig. 2 , and then gradually increase.

Figure 3 shows the stress-strain curves for the polyethylene matrix with armchair $(18,18)$ CNTs at three volume fractions, $f=1 \%, 5 \%$ and $10 \%$. The peak strengths for different $f$ are exactly the same, $406 \mathrm{MPa}$, such that the CNT volume fraction has no effect on the composite strength. The critical strain at the peak strength changes slightly, $13.9 \%$ for $f=10 \%, 14.6 \%$ for $f=5 \%$, and $15.2 \%$ for $f=1 \%$. The CNT volume fraction $f=10 \%$ gives a higher initial modulus than $f=5 \%$ (or 1\%), but it also gives a lower incremental modulus at large strain (after CNTs are completely debonded). For the displacement-controlled experiment, the vertical stress drop is $117 \mathrm{MPa}$ for $f=10 \%$, and $68 \mathrm{MPa}$ and $16 \mathrm{MPa}$ for $f=5 \%$ and $1 \%$, respectively. Therefore, CNTs may reinforce the composite at the small strain but weaken it at the large strain.

Figure 4 shows the effect of CNT size on the stress-strain curve. The CNT volume fraction is fixed at $f=10 \%$. Three different armchair CNTs, $(18,18),(12,12)$ and $(6,6)$, are examined, and the corresponding radii are $R=1.25,0.83$ and $0.42 \mathrm{~nm}$, respectively. The peak strength increases from $406 \mathrm{MPa}$ to 419 and $462 \mathrm{MPa}$ as the CNT radius decreases, from $(18,18)$ to $(12,12)$ and $(6,6)$. The critical strain for the peak strength also increases, from $13.9 \%$ to $14.6 \%$ and $16.8 \%$. Small CNTs clearly give stronger reinforcing effect than large CNTs because, at a fixed CNT volume fraction, there are more small CNTs than large ones, and therefore more interfaces. This observation of strong reinforcing effect for small CNTs also holds after CNTs 
are debonded from the matrix. The stress drop is $117 \mathrm{MPa}, 106 \mathrm{MPa}$ and $41 \mathrm{MPa}$ for the $(18,18)$, $(12,12)$, and $(6,6)$ CNTs. Therefore, small CNTs are more effective than large CNTs.

In order to investigate whether the interface bonding has a strong influence on the macroscopic behavior of CNT-reinforced composites, we double and quadruple the total cohesive energy to $\Phi_{\text {total }}=0.214 \mathrm{Jm}^{-2}$ and $0.429 \mathrm{Jm}^{-2}$ while maintaining the same cohesive strength $\sigma_{\max }=479 \mathrm{MPa}$. Figure 5 shows the stress-strain curve of the composite with $f=10 \%$ volume fraction of armchair $(18,18) \mathrm{CNTs}$. The peak strength increases from 406MPa to 432 and $496 \mathrm{MPa}$ when the total cohesive energy changes from $0.107 \mathrm{Jm}^{-2}$ to 0.214 and $0.429 \mathrm{Jm}^{-2}$, respectively. The corresponding strain for the peak strength also increases from $13.9 \%$ to $15.3 \%$ and $18.9 \%$. The overall stress-strain curve for large $\Phi_{\text {total }}\left(0.429 \mathrm{Jm}^{-2}\right)$ becomes much higher than that for low $\Phi_{\text {total }}\left(0.107 \mathrm{Jm}^{-2}\right)$. Therefore, strong adhesion between CNTs and polymer matrix may significantly improve the mechanical behavior of CNT-reinforced composites.

\section{Concluding Remarks}

For carbon nanotubes not well bonded to polymers, Jiang et al. [2] established a cohesive law for carbon nanotube/polymer interfaces. The cohesive law and its properties (e.g., cohesive strength, cohesive energy) are obtained directly from the Lennard-Jones potential from the van der Waals interactions. Such a cohesive law is incorporated in the micromechanics model to study the mechanical behavior of carbon nanotube-reinforced composite materials.

Carbon nanotubes indeed improve the mechanical behavior of composite at the small strain. However, such improvement disappears at relatively large strain because the completely debonded nanotubes behave like voids in the matrix and may even weaken the composite. The increase of interface adhesion between carbon nanotubes and polymer matrix may significantly improve the composite behavior at the large strain.

\section{Acknowledgement}

This research was supported by the ONR Composites for Marine Structures Program (grants N00014-01-1-0205, Program Manager Dr. Y. D. S. Rajapakse). 


\section{References}

1. Thostenson ET, Chou TW. Aligned multi-walled carbon nanotube-reinforced composites: processing and mechanical characterization. J Phys D: Appl Phys 2002;35:L77-L80.

2. Jiang LY, Huang Y, Jiang H, Ravichandran G, Gao H, Hwang KC, Liu B. A cohesive law for carbon nanotube/polymer interfaces based on the van der Waals force. J Mech Phys Solids 2006;54:2436-52.

3. Baughman RH, Zakhidov AA, de Heerm WA. Carbon nanotubes--the route toward applications. Science 2002;297:787-92.

4. Thostenson ET, Ren ZF, Chou TW. Advances in the science and technology of carbon nanotubes and their composites: a review. Compos Sci Technol 2001;61:1899-912.

5. Thostenson ET, Li CY, Chou TW. Nanocomposites in context. Compos Sci Technol $2005 ; 65: 491-516$.

6. Maruyama B, Alam H. Carbon nanotubes and nanofibers in composite materials. SAMPE J 2002;38:59-70.

7. Deepak S, Wei C, Cho K. Nanomechanics of carbon nanotubes and composites. Appl Mech Rev 2003;56:215-30.

8. Breuer O, Sundararaj U. Big returns from small fibers: a review of polymer/carbon nanotube composites. Polym Compos 2004;25:630-45.

9. Harris PJF. Carbon nanotube composites. Int Mater Rev 2004;49:31-43.

10. Schadler LS, Giannaris SC, Ajayan PM. Load transfer in carbon nanotube epoxy composites, Appl Phys Lett 1998;73:3842-4. 
11. Ajayan PM, Schadler LS, Giannaris C, Rubio A. Single-walled carbon nanotube-polymer composites: strength and weakness. Adv Mater 2000;12:750-3.

12. Lau KT, Shi SQ. Failure mechanisms of carbon nanotube/epoxy composites pre-treated in different temperature environments. Carbon 2002;40:2965-8.

13. Liao K, Li S. Interfacial characteristics of a carbon nanotube-polystyrene composite system. Appl Phys Lett 2001;79:4225-7.

14. Frankland SJV, Caglar A, Brenner DW, Griebel M. Molecular simulation of the influence of chemical cross-links on the shear strength of carbon nanotube-polymer interfaces. J Phys Chem B 2002;106:3046-48.

15. Li CY, Chou TW. Multiscale modeling of carbon nanotube reinforced polymer composites. J. Nanosci. Nanotechno 2003;3:423-30.

16. Wong M, Paramsothy M, Xu XJ, Ren Y, Li S, Liao K. Physical interactions at carbon nanotube-polymer interface. Polymer 2003;44:7757-64.

17. Gou J, Minaie B, Wang B, Liang Z, Zhang C. Computational and experimental study of interfacial bonding of single-walled nanotube reinforced composites. Comp Mater Sci 2004;31: 225-36.

18. Frankland SJV, Harik VM, Odegard GM, Brenner DW, Gates TS. The stress-strain behavior of polymer-nanotube composites from molecular dynamics simulation. Compos Sci Technol 2003;63:1655-61.

19. Jiang H, Zhang P, Liu B, Huang Y, Geubelle PH, Gao H, Hwang KC. The effect of nanotube radius on the constitutive model for carbon nanotubes. Comput Mater Sci 2003;28:429-42. 
20. Benveniste Y, Aboudi J. A continuum model for fiber reinforced materials with debonding. Int J Solids Struct 1984;20:935-51.

21. Tan H, Huang Y, Liu C, Geubelle PH. The Mori-Tanaka method for composite materials with nonlinear interface debonding. Int J Plasticity 2005;21:1890-918.

22. Timoshenko S, Goodier JN. Theory of elasticity, $3^{\text {rd }}$ ed. McGraw-Hill; 1970.

23. Reuss A. Berechung der Fliessgrenze von Mischkristallenx auf Grund der Plastizitätsbedingung für Einkristalle. Z Angew Math Mech 1929;9:49-58. 


\section{Figure captions}

Figure 1. The cohesive law for a carbon nanotube and polymer matrix established from the van der Waals interactions at the nanotube/matrix interface. The normal cohesive stress $\sigma^{\text {int }}$ is shown versus the interface opening displacement $[u]$ for a carbon nanotube and polyethylene matrix.

Figure 2. The macroscopic stress-strain relation of a carbon nanotube-reinforced polyethylene matrix composite subjected to hydrostatic tension. The volume fraction of armchair $(18,18)$ carbon nanotubes is $10 \%$. The linear elastic stress-strain relation of the polyethylene matrix is also shown.

Figure 3. The macroscopic stress-strain relation of a carbon nanotube-reinforced polyethylene matrix composite. The volume fraction of armchair $(18,18)$ carbon nanotubes is $10 \%, 5 \%$ and $1 \%$.

Figure 4. The macroscopic stress-strain relation of a carbon nanotube-reinforced polyethylene matrix composite. The volume fraction is $10 \%$ for three different armchair carbon nanotubes, $(18,18),(12,12)$ and $(6,6)$.

Figure 5. The macroscopic stress-strain relation of a carbon nanotube-reinforced polyethylene matrix composite. The volume fraction is $10 \%$ for armchair $(18,18)$ carbon nanotubes. Three values of the total cohesive energy are taken, $\Phi_{\text {total }}=0.107,0.214$ and $0.429 \mathrm{Jm}^{-2}$. The cohesive strength is fixed at $479 \mathrm{MPa}$. 
Figure 1

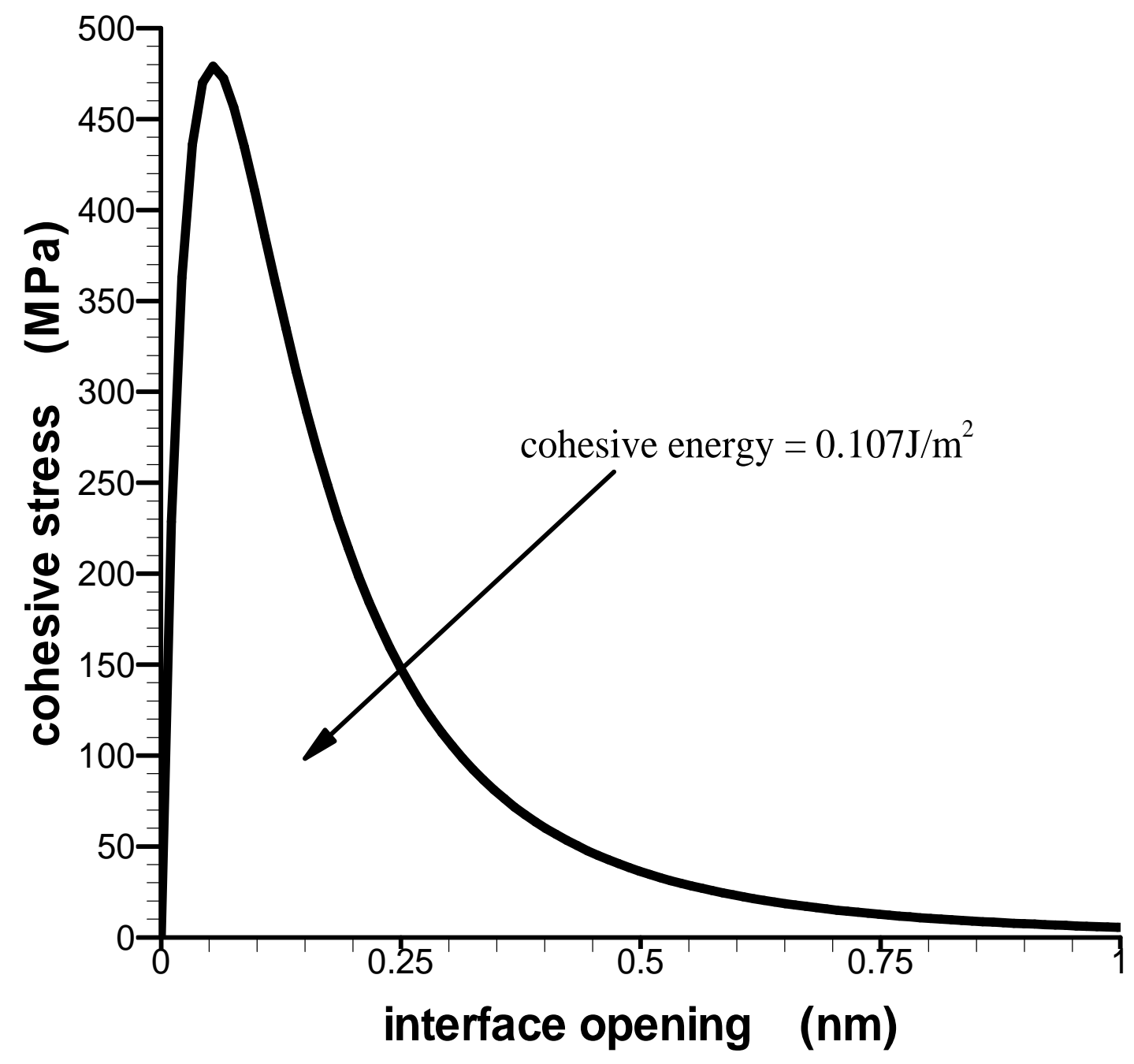


Figure 2

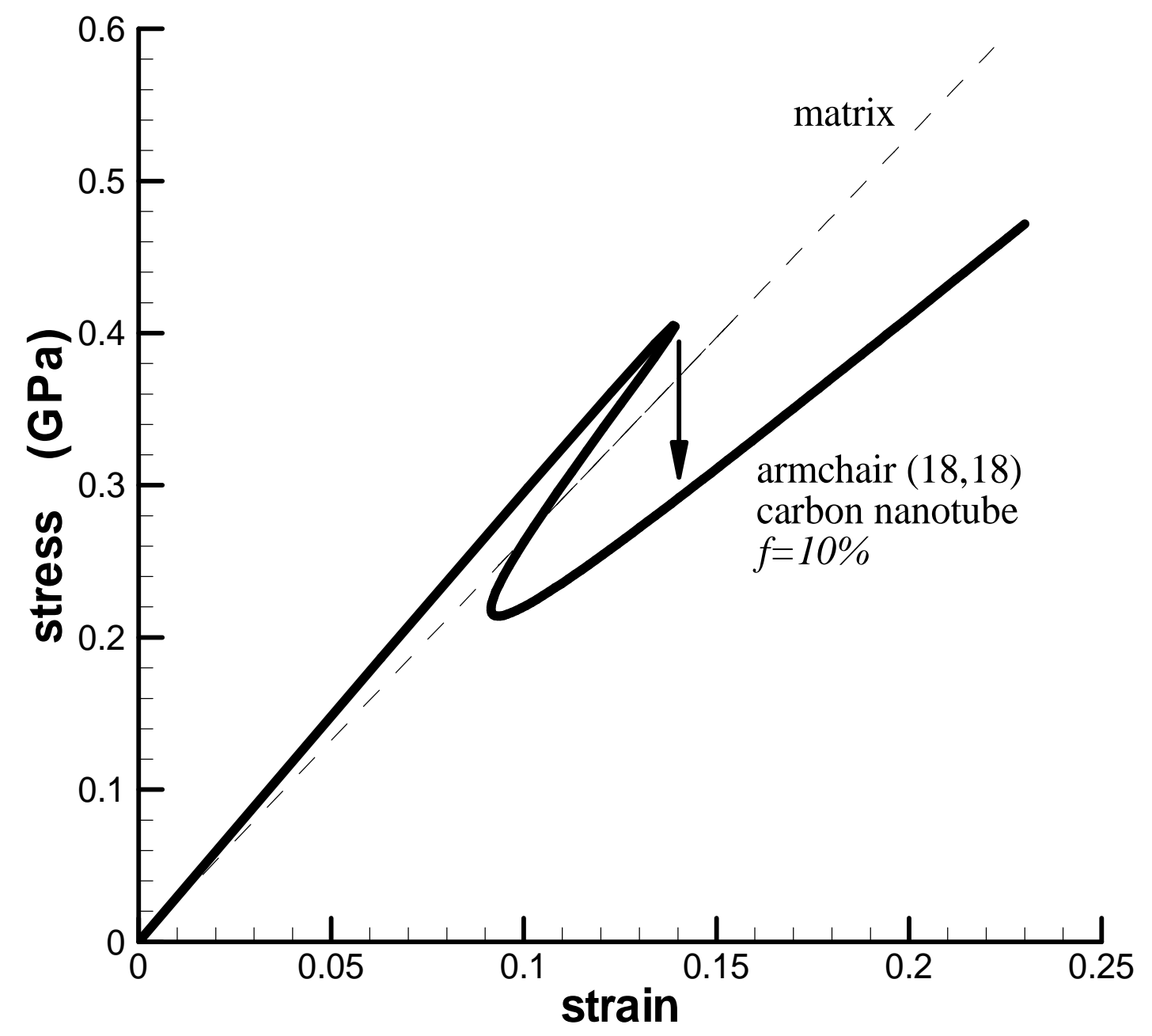


Figure 3




Figure 4

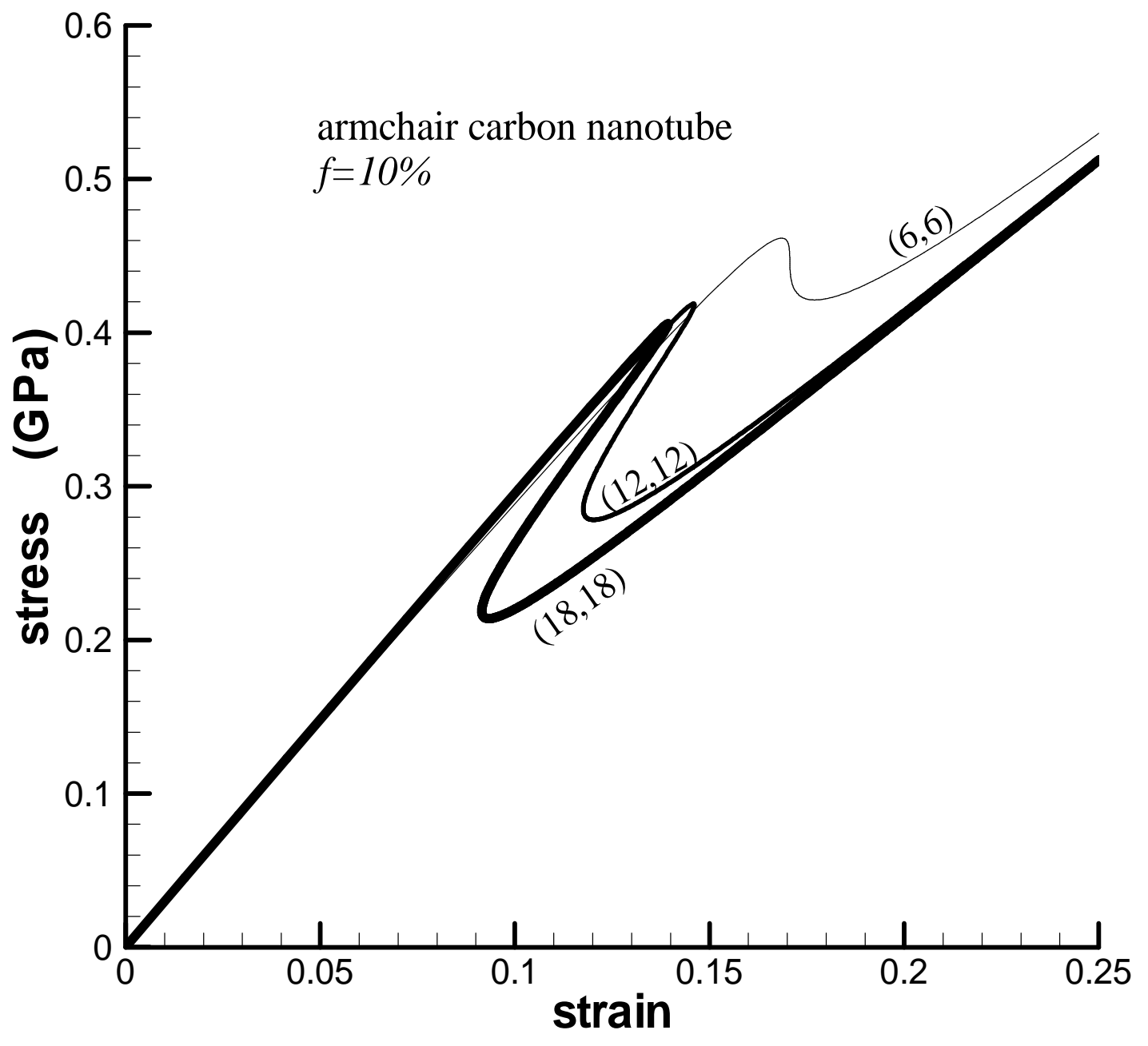




\section{Figure 5}

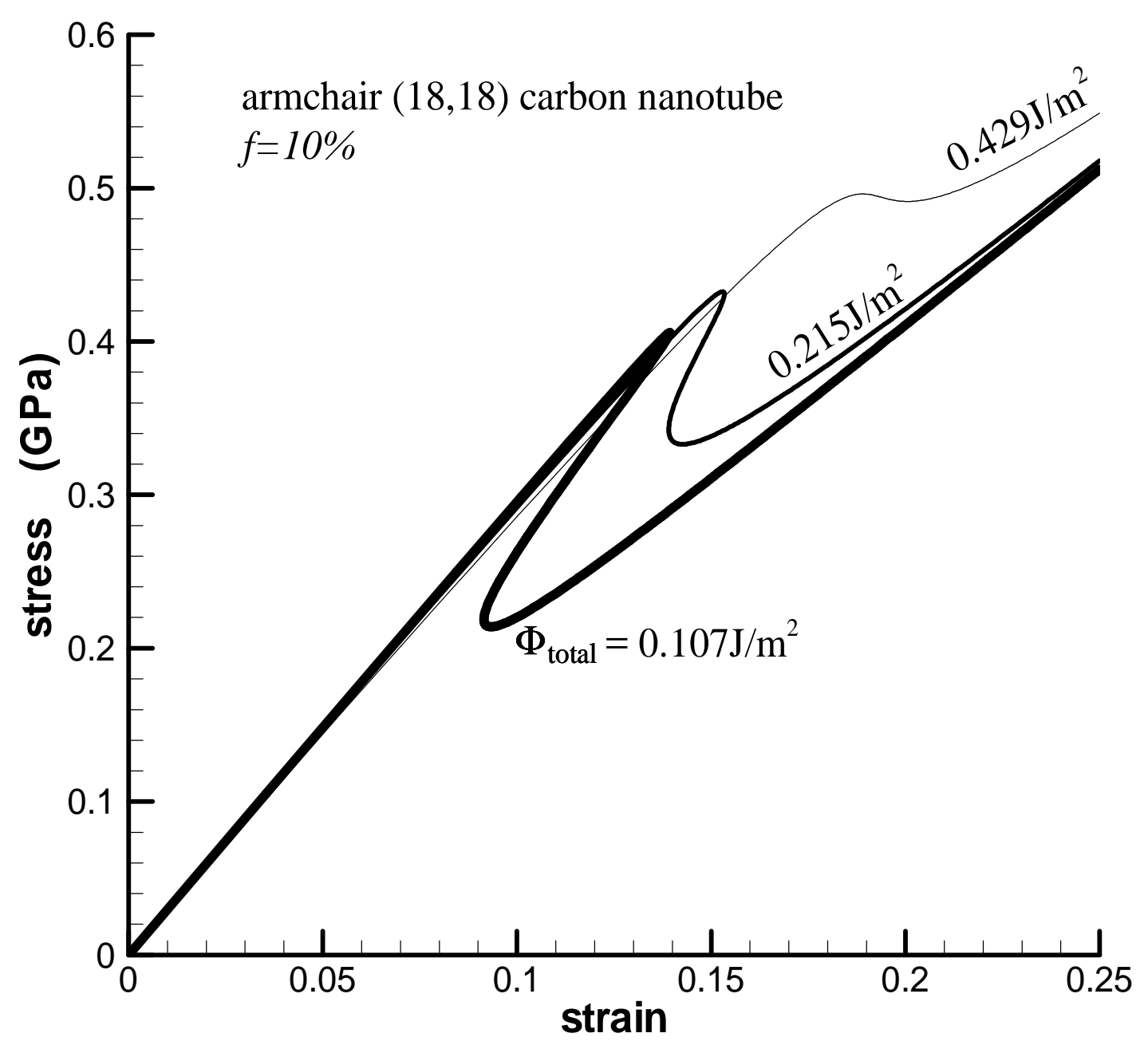

